\title{
Using noble gas diffusion kinetics to inform geochronology
}

\author{
MARISSA M. TREMBLAY ${ }^{1}$
}

${ }^{1}$ Earth, Atmospheric, and Planetary Sciences, Purdue University, 550 Stadium Mall Drive, West Lafayette, IN

47901, USA; tremblam@purdue.edu

In geochronology, we often study geologic problems that enable simplifying assumptions about our chronometers. For example, if a lava flow cools over tens of years to surface temperatures, we can assume that the minerals in the lava became closed to argon (Ar) diffusion instantaneously, or at least within the resolution of the ${ }^{40} \mathrm{Ar} /{ }^{39} \mathrm{Ar}$ chronometer. This is distinct from thermochronology, in which we consider geologic problems where diffusion kinetics are essential to interpreting chronological results. However, there are cases where noble gas diffusion kinetics become relevant even for problems conventionally in the realm of geochronology. In these cases, utilizing information about noble gas diffusion kinetics often provides a deeper understanding of the geologic problem at hand. In this talk, I will highlight three examples where the knowledge of noble gas diffusion kinetics is paramount to how we interpret geochronological datasets.

First, I will discuss ${ }^{40} \mathrm{Ar} /{ }^{39} \mathrm{Ar}$ data from a suite of Miocene ignimbrites that show dispersion in sanidine ${ }^{40} \mathrm{Ar} /{ }^{39} \mathrm{Ar}$ ages of several million years. I will demonstrate how we can use sanidine Ar diffusion kinetics along with this age dispersion to constrain the storage temperatures and magmatic residence times for these ignimbrites.

Second, I will discuss ${ }^{40} \mathrm{Ar} /{ }^{39} \mathrm{Ar}$ ages from iddingsite sampled from the Martian meteorite Lafayette, which is interpreted to date when there was liquid water near the surface of Mars. This age interpretation requires that no ${ }^{40} \mathrm{Ar}$ diffusion occurred during the impact ejection, space transit, and atmospheric entry of Lafayette. I will demonstrate how we tested we tested whether these events could have compromised the Lafayette iddingsite ${ }^{40} \mathrm{Ar} /{ }^{39} \mathrm{Ar}$ record using Ar diffusion kinetics from an analog material.

Third, I will discuss a new approach to deconvolving ${ }^{40} \mathrm{Ar} /{ }^{39} \mathrm{Ar}$ datasets from mixtures, which are often necessary but also advantageous to collect for studies of planetary materials and fine-grained terrestrial detrital samples. I will describe a Bayesian modeling framework for deconvolving the ages of individual components in an ${ }^{40} \mathrm{Ar} /{ }^{39} \mathrm{Ar}$ dataset from a mixture, and highlight how variations in Ar diffusion kinetics in different minerals lies at the heart of this model framework. 